\title{
Reactive mesothelial proliferation: a necropsy study
}

\author{
CD SHELDON, AMANDA HERBERT, PJ GALLAGHER
}

From the Department of Pathology, Southampton University General Hospital, Southampton

ABSTRACT To establish a histological standard against which surgical biopsy material could be compared, the degree of mesothelial proliferation was studied in 100 unselected necropsies. A minor degree of mesothelial hyperplasia was identified in 10 cases, usually close to areas of fibrous adhesions. Pleural plaques were present in 33 patients but there was no evidence of associated mesothelial proliferation. No mesothelial changes were noted in patients with empyema or pleural metastases. These findings indicate that the degree of mesothelial hyperplasia in common disorders of the pleura is relatively slight. Significant mesothelial proliferation in needle biopsies should therefore be viewed with considerable suspicion and, where clinically appropriate, be followed by further investigation.

The extensive use of asbestos in the Southampton area over a period of at least 25 years has led to a high local incidence of pulmonary and pleural fibrosis and malignant mesothelioma. Over 150 pleural biopsies are examined in our surgical pathology laboratory annually but the evaluation of mesothelial proliferation is a recurrent histological problem. Although cytological examination of pleural fluid is often useful in the investigation of pulmonary carcinoma, ${ }^{3}$ the cytological diagnosis of malignant mesothelioma from pleural aspirates is notoriously difficult. 6 7

We were primarily concerned that mesothelial proliferation associated with common disorders such as fibrous adhesions, pleural plaques, or empyemata might be a source of diagnostic confusion. To provide a standard against which surgical biopsy material might be compared we have made a detailed histological study of the mesothelium in 100 unselected necropsies.

\section{Methods}

The pleural and peritoneal cavities, the lungs and visceral pleura, both surfaces of the diaphragm, and the splenic capsule were examined in 100 necropsies. The number, size, and position of any lesions were recorded and samples of normal and abnormal tissue taken for histology. Three levels of a block of the right lower lobe of the lung were cut at $15 \mu \mathrm{m}$ and examined unstained for asbestos bodies.

Address for reprint requests: Dr PJ Gallagher, Department of Pathology, Southampton University General Hospital, Southampton SO9 4XY.
Using a four-point scale (nil, slight, moderate, and marked) the degree of mesothelial hyperplasia, vasodilatation and fibrosis was assessed in both the visceral and parietal pleural sections of each case. In addition a record was made of the nature and density of any associated inflammatory infiltrates.

\section{Results}

The causes of death of the patients studied are shown in table 1. The mean age of the 64 men was 66.6

Table 1 Causes of death of patients studied

\begin{tabular}{lc}
\hline Cause of death & Number of patients \\
\hline Ischaemic heart disease & 23 \\
Chronic cardiac failure & 12 \\
Other cardiovascular causes & 8 \\
Respiratory tract infections & 14 \\
Pulmonary embolism & 6 \\
Bronchial carcinoma & 7 \\
Tumours other than bronchial carcinoma & 8 \\
Renal disease & 5 \\
Cerebrovascular accidents & 4 \\
Septicaemia & 4 \\
Trauma & 3 \\
Others & 6 \\
\hline
\end{tabular}

Table 2 Frequency of macroscopic changes in pleural cavities and splenic capsules

\begin{tabular}{ll}
\hline Macroscopic change & Frequency (\%) \\
\hline Pleural plaques & 33 \\
Fibrous pleural adhesions & 22 \\
Areas of pleural fibrosis (not plaques) & 10 \\
Inflammatory changes & 14 \\
Secondary tumour deposits & 8 \\
Hyalinised areas on splenic capsule & 13 \\
No macroscopic abnormalities & 33 \\
\hline
\end{tabular}


years (range 26-98 yr) and the 36 women $73 \cdot 1$ years (range 24-95 yr).

\section{MACROSCOPIC FINDINGS}

The changes identified are summarised in table 2 . In 33 cases no abnormalities were detected. Fibrous plaques were present in 30 men and three women. They were most frequent in the posterolateral portions of the chest wall and tended to follow the surfaces of the lower five ribs more than the intercostal spaces. Lesions ranged from $0.5 \times 0.5 \mathrm{~cm}$. to $10 \times 12 \mathrm{~cm}$. Of the 33 patients with pleural plaques 17 had plaques alone, 12 plaques and fibrous adhesions, seven hyalinised areas on the splenic capsule, three associated pleural inflammation, and one metastatic carcinomatous deposits. There was no significant statistical association between the presence of pleural plaques and fibrous adhesions $\left(\chi^{2}=\right.$ $2 \cdot 6, p>0 \cdot 1)$ or pleural plaques and hyalinised areas on the splenic capsule $\left(\chi^{2}=0.003, p>0.5\right)$.

Fourteen patients had macroscopic evidence of pleural inflammation. In one a large empyema had

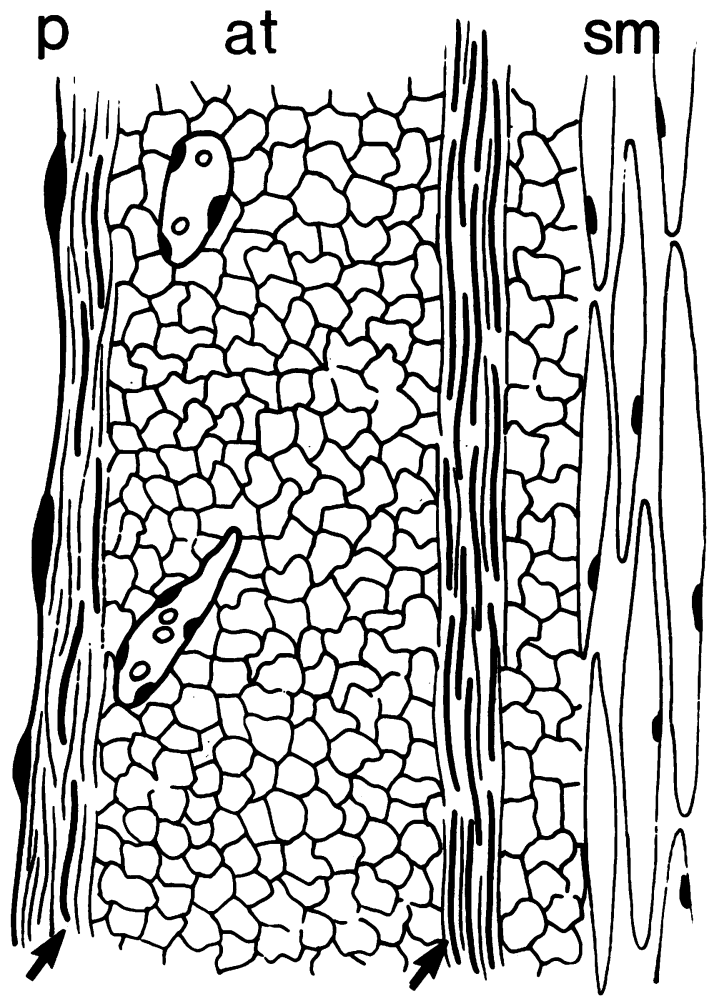

Fig 1 Diagrammatic representation of the structure of normal parietal pleura (see text for details). p: parietal pleura, arrows: connective tissue bands (thin lines collagen, thick lines elastic), at: adipose tissue, sm: skeletal muscle.

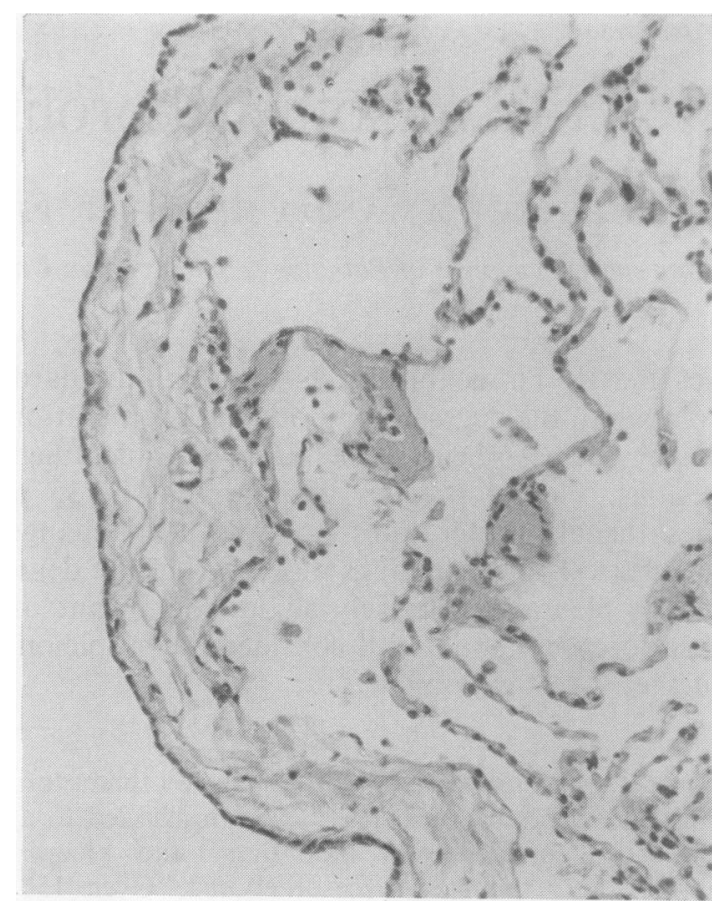

Fig 2 Normal visceral pleura. The single layered mesothelium is applied to a thin band of fibrous tissue. $H$ and $E, \times 180$.

formed and in another extensive bilateral fibrinous exudates were present in both pleural cavities. The changes in the remaining 12 cases were relatively slight.

\section{MICROSCOPIC CHANGES}

Normal pleural cavities

Normal pleural histology is illustrated in figs 1 and 2. The mesothelium is present as a single layer in all portions of the thoracic cavity and in many sections is barely discernible. A band of compressed connective tissue, up to $100 \mu \mathrm{m}$ in thickness, separates the mesothelium from the underlying adipose tissue. Between the adipose tissue layer and the intercostal skeletal muscles is a further compressed band of connective tissue (usually 80-120 $\mu \mathrm{m}$ ) containing relatively more elastic tissue than the submesothelial layer.

\section{Mesothelial proliferation}

In $90 \%$ of cases the mesothelium was entirely normal. Mesothelial hyperplasia was identified in 10 cases (seven men, three women; mean age $71.3 \mathrm{yr}$, range 62-87). In eight of these the hyperplastic changes were seen close to areas of pleural fibrosis or fibrous pleural adhesions. Even in the most florid 


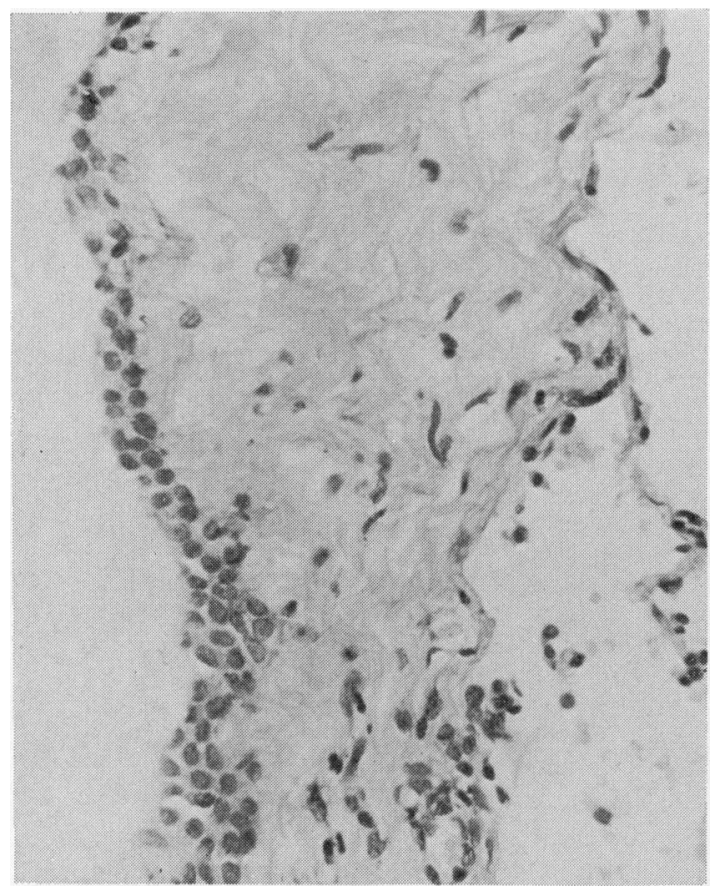

Fig 3 Slight hyperplasia of visceral pleural mesothelium close to an area of pleural fibrosis. $H$ and $E, \times 245$.

cases (figs 3 and 4) there was only slight nuclear pleomorphism, and although nucleoli were occasionally conspicuous (fig 4) no mitoses were seen. The mesothelium rarely exceeded two layers in thickness even in areas close to fibrous adhesions (fig 4). Multilayering could usually be attributed to histological artefact.

\section{Pleural plaques}

The idealised structure of a pleural plaque is shown in fig 5 . In the vast majority of the plaques sectioned the mesothelial covering was incomplete. There was no evidence of mesothelial hyperplasia or regeneration, even in the three cases in which the mesothelium was intact. Occasional clusters of mixed chronic inflammatory cells were present at the periphery of plaques. The central collagenous core was relatively acellular and generally had a characteristic basket weave appearance. Plaque thickness varied between 0.3 and $9.0 \mathrm{~mm}$ (mean $2.5 \mathrm{~mm}$ ). Plaques greater than about $3 \mathrm{~mm}$ in thickness often had focal areas of calcification.

\section{Asbestos bodies}

In 17 patients one or more asbestos bodies were

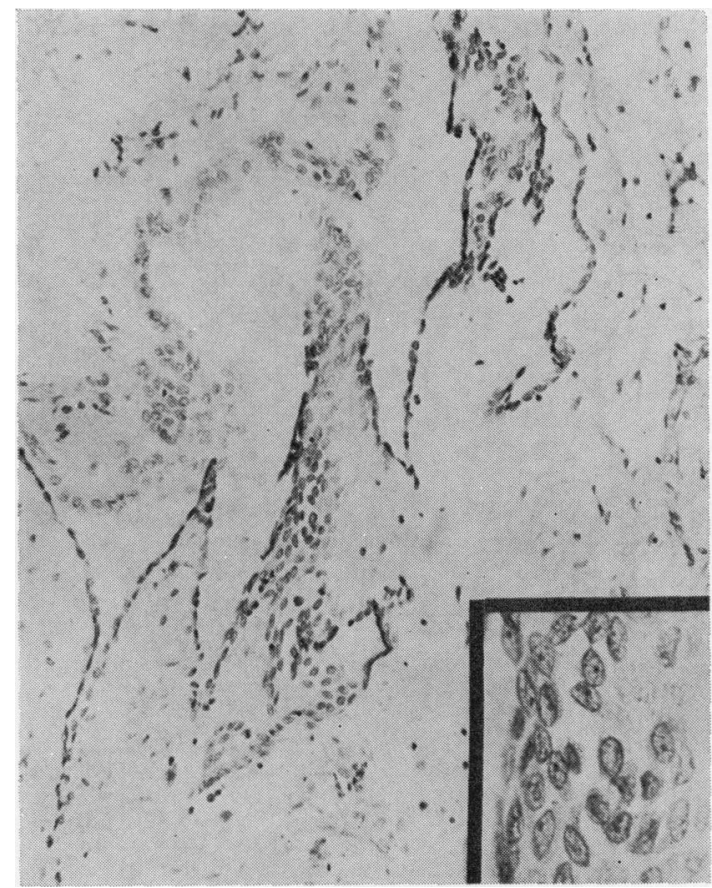

Fig 4 Hyperplasia of parietal pleural mesothelium in an area of pleural fibrosis. Some of the multilayering can be attributed to histological cutting artefact. $H$ and $E$, $\times$ 125. Inset: conspicuous nucleoli of hyperplastic mesothelial cells. $H$ and $E, \times 500$.

identified in the sections of the right lower lobe. In 11 of these cases pleural plaques were present, three had areas of pleural fibrosis, one a primary bronchial carcinoma, one pleural metastatic deposits, and one a normal pleural cavity. There was a significant association between pleural plaques and the presence of asbestos bodies $\left(\chi^{2}=7 \cdot 7, \mathrm{p}<0.01\right)$. There was an occupational history of asbestos exposure in two of the 10 male patients, but none of the seven women.

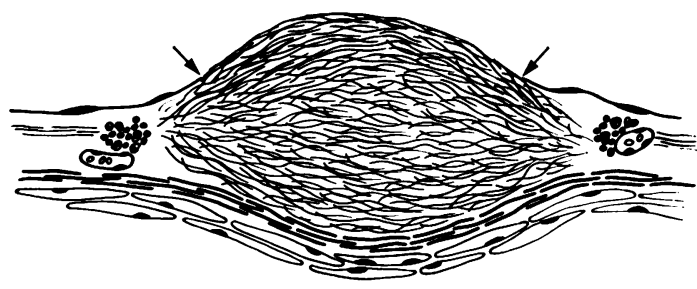

Fig 5 Diagrammatic representation of structure of a pleural plaque (see text for details). Note central mass of dense connective tissue protruding through mesothelial covering (arrows). 


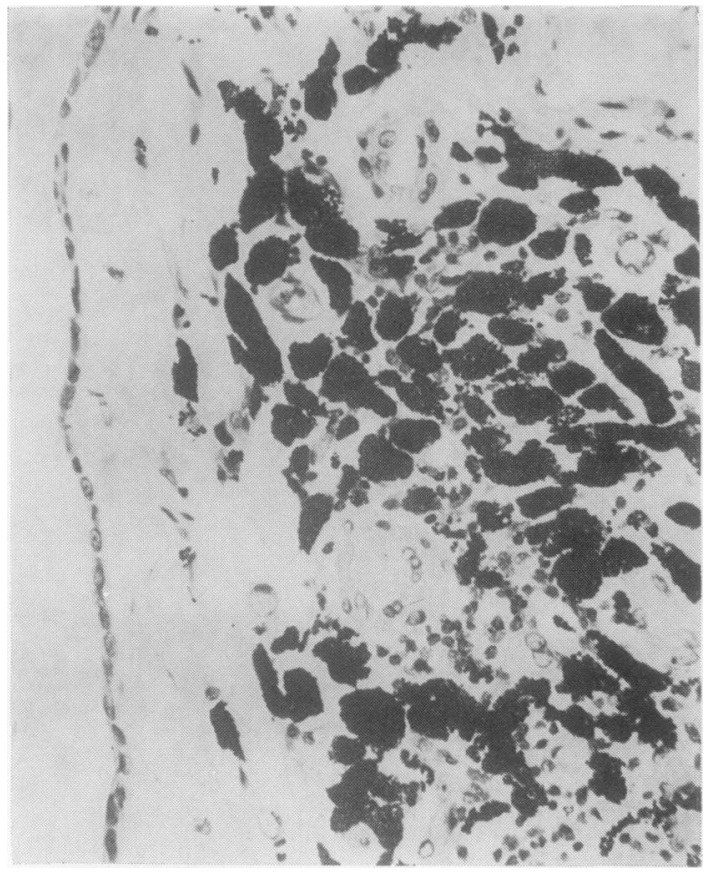

Fig 6 Parietal pleural dust deposits. No associated mesothelial changes. $H$ and $E, \times 280$.

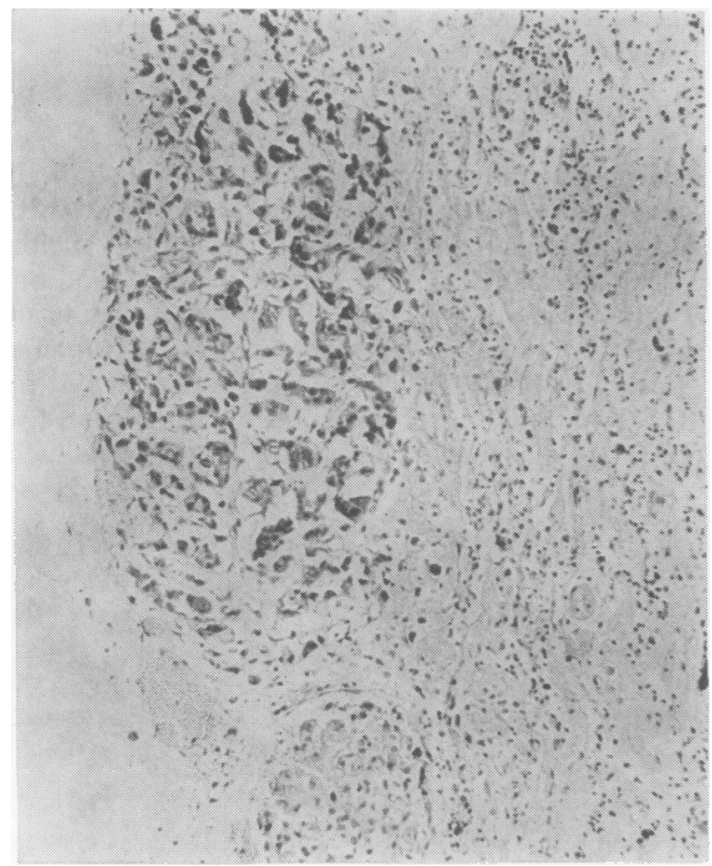

Fig 7 Parietal pleural deposit of metastatic bronchial carcinoma. The mesothelial covering is barely discernible. $H$ and $E, \times 145$.

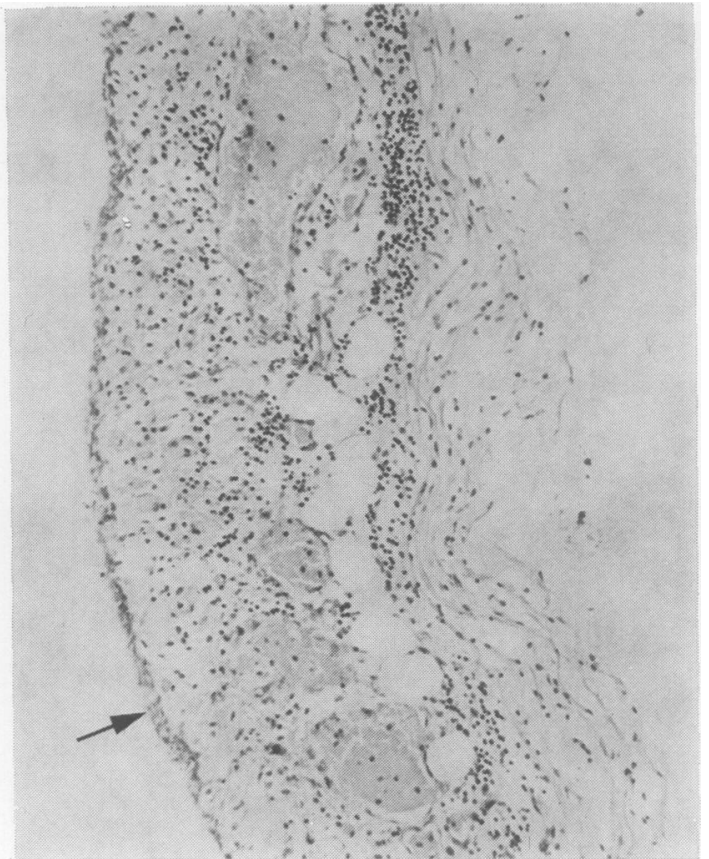

Fig 8 Parietal pleura of a patient with an empyema. The mesothelium is partially necrotic (arrow). $H$ and $E$, $\times 90$.

\section{Other microscopic changes}

No mesothelial abnormalities were detected around tumour deposits or areas of pleural dust pigmentation (figs 6 and 7). Acute inflammatory changes were present in the pleura of 10 patients and chronic inflammation in six. When inflammation was extensive the mesothelium was often absent or necrotic (figs 8 and 9). A minor degree of mesothelial hyperplasia was identified in one patient only.

\section{Discussion}

The purpose of this study was to define the degree of mesothelial proliferation that occurs in common disorders of the pleural cavity. A clear-cut result was obtained. Florid mesothelial proliferation was never recognised. A minor degree of hyperplasia was identified in $10 \%$ of cases, usually close to areas of pleural fibrosis.

In any investigation of this nature it is important to consider whether the patients studied were representative of the population that might be found in the relevant clinical setting. Our cases, like those seen in most pulmonary units, were predominantly male, and were elderly. Furthermore the incidence of common macroscopic abnormalities, such as pleural plaques, was similar to other and sometimes larger necropsy surveys. ${ }^{2} 510$ 


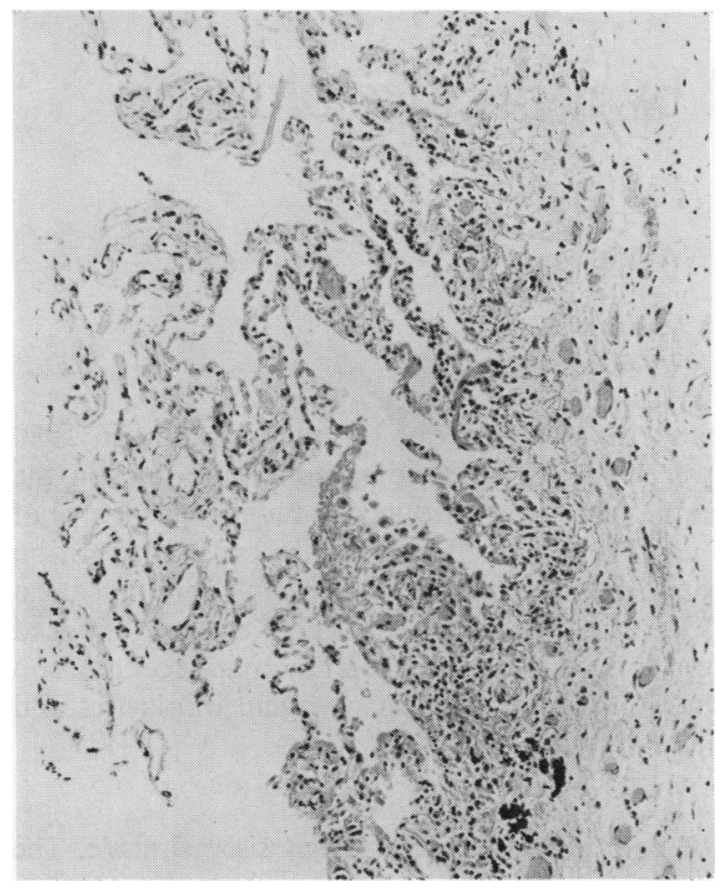

Fig 9 Parietal pleura close to a fibrous adhesion. Many fibrinous strands have formed but there is no associated mesothelial hyperplasia. $H$ and $E, \times 125$.

Pleural biopsy often enables a diagnosis of pulmonary carcinoma to be firmly established. In two recent series the rate of positive diagnosis in biopsies from patients subsequently shown to have pulmonary carcinoma at operation or necropsy was $48 \%$ and $70 \%{ }^{111}$ Our own experience is that the diagnosis of malignant mesothelioma from needle biopsies of the pleura is far more difficult than that of bronchial carcinoma. Klima and Gyorkey emphasised that binucleation and hyperchromaticity were consistent findings in the mesothelium of patients who were later shown to have malignant mesothelioma. ${ }^{4}$ However Rosai and Dehner reported a wide variety of histological abnormalities, in- cluding mitotic activity and multinucleation, in a series of 13 cases of mesothelial hyperplasia in abdominal hernial sacs. ${ }^{8}$ Despite this report we consider that the results of our study, coupled with the findings of Klima and Gyorkey, ${ }^{4}$ indicate that mesothelial changes such as nuclear hyperchromatism, atypia, or mitotic activity must not be lightly dismissed. They are unlikely to occur in nonneoplastic disorders of the pleura. Such patients, particularly those with an occupational history of asbestos exposure, or an unusual pattern of pulmonary illness should be rebiopsied, and appropriate further investigation or follow-up planned.

We thank the Department of Teaching Media, University of Southampton for preparing the artwork and Miss Margaret Harris for typing the manuscript.

\section{References}

${ }^{1}$ Deluccia VC, Reyes EC. Percutaneous needle biopsy of parietal pleura. Analysis of 50 cases. NY State J Med 1977;77:2058-61.

${ }^{2}$ Francis D, Jussey A, Mortensen T, Sikjaer B, Viskum K. Hyaline pleural plaques and asbestos bodies in 198 randomised autopsies. Scand J Respir Dis 1977;58: 193-6.

${ }^{3}$ Frist B, Kahan AV, Koss LG. Comparison of the diagnostic values of biopsies of the pleura and cytologic evaluations of pleural fluids. Am J Clin Pathol 1979;72:48-51.

${ }^{4}$ Klima M, Gyorkey F. Benign pleural lesions and malignant mesothelioma. Virchows Arch 1977;376:181-93.

5 Meurman L. Asbestos bodies and pleural plaques in a Finnish series of autopsy cases. Acta Pathol Microbiol Scand 1966; Supplement 181.

- Naylor B. The exfoliative cytology of diffuse malignant mesothelioma. J Pathol Bacteriol 1963;86:293-8.

' Roberts GH, Campbell GM. Exfoliative cytology of diffuse mesothelioma. J Clin Pathol 1972;25:577-82.

${ }^{8}$ Rosai J, Dehner LP. Nodular mesothelial hyperplasia in hernia sacs. A benign reactive condition simulating a neoplastic process. Cancer $1975 ; 35: 165-75$.

? Rous V, Studeny J. Aetiology of pleural plaques. Thorax 1970;25:270-84.

${ }^{10}$ Robinson JJ. Pleural plaques and splenic capsular sclerosis in adult male autopsies. Arch Pathol 1972;93:118-22.

11 Van Hoff DD, Li Volsi V. Diagnostic reliability of needle biopsy of the parietal pleura. A review of 272 biopsies. Am J Clin Pathol 1975;64:200-3. 